TI 2017-093/V

Tinbergen Institute Discussion Paper
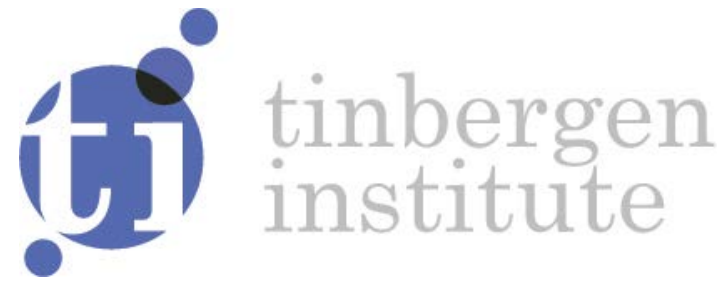

\title{
Artificial Pitches and Unfair Home Advantage in Professional Football
}

Jan C. van Ours ${ }^{1}$

${ }^{1}$ Erasmus School of Economics, Erasmus University Rotterdam; Tinbergen Institute, The Netherlands 
Tinbergen Institute is the graduate school and research institute in economics of Erasmus University Rotterdam, the University of Amsterdam and VU University Amsterdam.

Contact: discussionpapers@tinbergen.nl

More TI discussion papers can be downloaded at http://www.tinbergen.nl

Tinbergen Institute has two locations:

Tinbergen Institute Amsterdam

Gustav Mahlerplein 117

1082 MS Amsterdam

The Netherlands

Tel.: $+31(0) 205984580$

Tinbergen Institute Rotterdam

Burg. Oudlaan 50

3062 PA Rotterdam

The Netherlands

Tel.: +31(0)10408 8900 


\title{
Artificial Pitches and Unfair Home Advantage in Professional Football
}

\author{
Jan C. van Ours*
}

September 28, 2017

\begin{abstract}
In the Netherlands, in the top tier of professional football some teams play their home matches on an artificial pitch while other teams play their home matches on natural grass. This paper investigates whether or not home teams who play on an artificial pitch have an additional home advantage to the regular home advantage. The main finding is, that this is indeed the case. This implies that artificial pitches generate an unfair home advantage in a competitive sport.
\end{abstract}

Keywords: Professional football, unfair competition, artificial pitch JEL code: Z21, L83

The author thanks Lucas Besters, Ruud Koning, Martin van Tuijl, Thomas Peeters and Thijs Velema for critical remarks on a previous version of the paper.

*Erasmus School of Economics, Erasmus University Rotterdam and Tinbergen Institute (Amsterdam/Rotterdam), The Netherlands; Department of Economics, University of Melbourne, Parkville, Australia and CEPR (London); vanours@ese.eur.nl. 


\section{Introduction}

In professional football, some teams play their home matches on an artificial pitch. There are often financial reasons for doing this, because maintenance costs are substantially lower than those of a natural grass pitch. However, artificial grass is not very popular among players and coaches. There are at least three reasons for this (Trombley (2016)). There is a perception of artificial grass increasing injury rates, being more tiring to play on and introducing a different behavior of the ball, which is thought to move faster and bounce higher than it does on natural grass. There is no evidence that playing on artificial grass increases injury rates (see, for example, Ekstrand et al. (2011) and Lanzetti et al. (2017)). An artificial pitch may be more tiring and it may introduce different ball dynamics, leading to a different playing style (Andersson et al. (2008)), but as long as these differences affect both teams equally, there is no reason for concern. Such a concern arises if a team playing on artificial grass has an additional home advantage compared to teams playing on natural grass. If so, an artificial pitch introduces an unfair home advantage in a highly competitive sport.

There is no dispute about the existence of a regular home advantage in professional football. This home advantage is mainly attributed to the support of the home crowd in combination with possibly biased referee decisions, players' familiarity with their stadium and travel fatigue of the away team (see, for example, Clarke and Norman (1995), Pollard (2006), Pollard and Armatas (2017) and Ponzo and Scoppa (2017)). Whether or not an artificial pitch introduces an additional home advantage has rarely been investigated. Barnett and Hilditch (1993) is one of the few studies that does this. The study investigates four teams in English league football (Luton Town, Oldham Athletic, Preston North End and Queen's Park Rangers) that had at least for some seasons an artificial pitch in the early 1980s. The main conclusion is that there is indeed an additional home advantage related to an artificial pitch equivalent to 0.28 points and 0.31 goals per match. In the meantime, the quality of artificial grass has improved substantially and the question is, whether current professional football teams that play on artificial grass still have an additional home advantage. Trombley (2016), analyzing four seasons (2011 to 2014) of US Major League Soccer, suggests that this is not the case. However, Hvattum (2015) concludes from an analysis of Norwegian league football from 2001 to 2013 that teams playing on artificial turf have a greater home advantage of approximately 2.5 points over the season.

The current paper investigates, whether teams who play their home matches on arti- 
ficial grass have an additional home advantage compared to teams who play their home matches on natural grass. To do this, data are used from the top tier of Dutch professional football, the "Eredivisie". The identification of the artificial grass effect is based on a simple strategy that takes into account that the difference in strength between two teams cancels out in pairs of matches.

\section{Data and Exploratory Analysis}

The Dutch top tier of professional football has 18 teams each of which plays 17 home and 17 away matches. For a win a team gets three points, for a draw this is one and for a loss no points are given. The team that has the highest number of points after 34 matches wins the championship and has traditionally been entitled to play in the European Champions League the next season. The team with the lowest number of points is relegated and replaced by the winner of the second tier. Number 16 and 17 play post-competition matches against teams from the second tier to determine whether or not they will be relegated.

The analysis is based on match results from three seasons, 2014-15 to 2016-17. In each of these seasons 6 teams had an artificial pitch whereas in earlier seasons only one or two teams had such a pitch. Present in all seasons were 14 teams, whereas four teams were present in two seasons and four teams in one season only (see Table 4 for an overview, also of the teams who have an artificial pitch). Figure 1 gives a summary overview at the level of team-season of the number of goals scored and the number of goals conceded by the end of the season. There is a clear negative relationship between goals scored and goals conceded. The strongest teams are bottom-right, the weakest teams are top-left. The teams with a natural grass pitch are distributed over the whole range while the teams with an artificial pitch are predominantly top-left. This is no coincidence. Teams with a smaller budget are more likely to be weaker and for financial reasons more likely to have an artificial pitch.

Table 1 presents a summary overview of home goals and away goals in matches between two teams both playing on natural grass and two teams of which one plays on an artificial pitch and the other plays on natural grass. Using the information presented in this table, the additional home advantage of playing on an artificial pitch can be established. The line of reasoning is as follows. If we look at all matches between teams who both play on natural grass, on average over the three seasons the home team scores 1.63 goals 
Figure 1: Goals scored and goals conceded by team; end of season 2014-15 2016-17

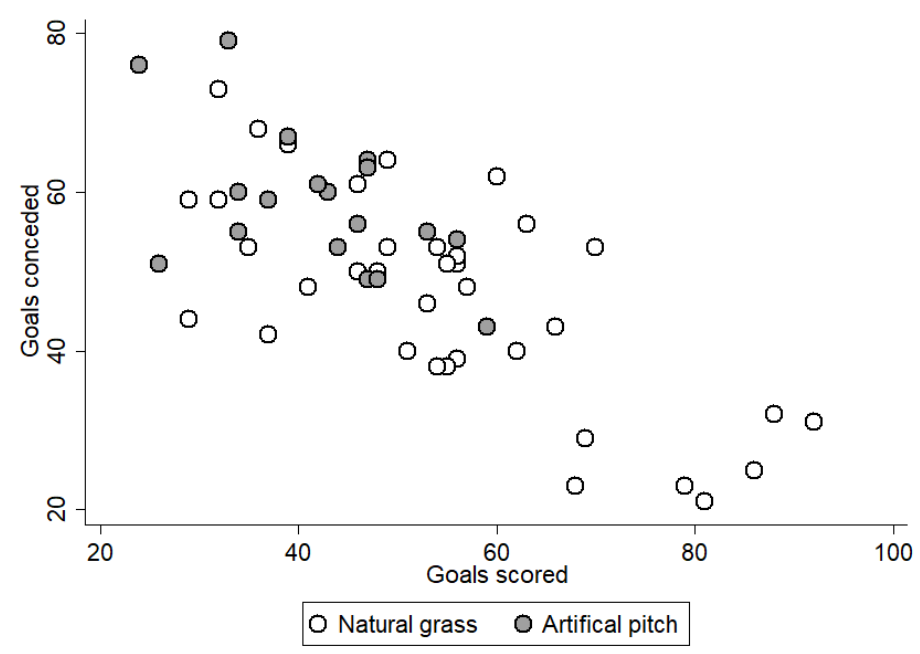

Note: there are 54 observations (18 teams in 3 seasons)

per match while the away team scores 1.42. Since, on average, over all matches, the difference in the strength between the two teams is equal, the goal difference of 0.21 can be attributed to the regular home advantage. Similarly, when two teams with a different home ground play against each other, the average number of goals scored in home matches is equal to 1.68, while the average number of away goals is equal to 1.20. The goal difference of 0.48 can be attributed to the regular home advantage and half of the additional home advantage related to playing on an artificial pitch (since half of these matches were played on an artificial pitch and half were played on natural grass). Therefore, twice the difference of the differences (equal to 0.53) represents the additional home advantage related to an artificial pitch.

Table 1: Goals, points and wins by type of match

\begin{tabular}{lcccccccccc}
\hline & \multicolumn{1}{c}{ Goals } & \multicolumn{1}{c}{ Points } & \multicolumn{1}{c}{ Win } \\
\cline { 2 - 10 } & $\mathrm{H}$ & $\mathrm{A}$ & $\Delta$ & $\mathrm{H}$ & $\mathrm{A}$ & $\Delta$ & $\mathrm{H}$ & $\mathrm{A}$ & $\Delta$ & Obs. \\
\hline Different pitch & 1.68 & 1.20 & 0.48 & 1.68 & 1.08 & 0.60 & 0.48 & 0.28 & 0.20 & 396 \\
Both natural grass & 1.63 & 1.42 & $\underline{0.21}$ & 1.53 & 1.23 & $\underline{0.30}$ & 0.43 & 0.33 & $\underline{0.10}$ & 432 \\
$\Delta \Delta$ & & & 0.27 & & & 0.30 & & & 0.10 & 828 \\
\hline AAP $\left(2^{*} \Delta \Delta\right)$ & & & 0.53 & & & 0.60 & & & 0.20 & \\
\hline
\end{tabular}

Note: $\mathrm{H}=$ home; $\mathrm{A}=$ away; $\Delta=$ Difference; AAP = extra advantage artificial pitch; obs. = number of observations. Different pitch: one team has natural grass, the other has an artificial pitch.

Table 1 also provides information about the average number of points achieved by 
home teams and away teams. Following the same line of reasoning, the regular home advantage in number of points is equal to 0.30 while the additional home advantage related to an artificial pitch is equal to $2^{*} 0.30=0.60$. Finally, Table 1 shows that on natural grass matches, home teams have a probability to win of $43 \%$ while this is $33 \%$ for away teams. Therefore, in term of win probability the regular home advantage is $10 \%$, while the additional win probability for teams who play their home matches on a natural pitch is $20 \%$.

\section{Statistical Model and Parameter Estimates}

The line of reasoning to establish the effects of an artificial pitch can be formalized as follows. Since the main interest is in the competitive disadvantage of natural grass teams when playing on artificial pitches, as in Table 1, I ignore matches between teams who both play on an artificial pitch. For all matches played at home by team $i$ against opponent team $j$ in season $t$ it holds (see also Clarke and Norman (1995)):

$$
y_{i j t}=\alpha_{i t}-\alpha_{j t}+\beta+d_{i} \gamma+\varepsilon_{i j t}
$$

where $y$ represents the indicator for the match results from the perspective of the home team, i.e. goal advantage, points advantage and win advantage. However, by using this approach there is a perfect correlation between points advantage and win advantage, i.e. if the home team wins, draws or loses the point difference is $+3,0$ and -3 respectively. The win advantage is $+1,0$ and -1 . Because of this perfect correlation, I ignore win advantage focusing on goals advantage and points advantage. Furthermore, in equation $(1), d_{i}$ is a dummy variable indicating whether or not team $i$ plays on artificial grass, $\alpha_{i}$ represents the strength of team $i$ and $\alpha_{j}$ represents the strength of team $j$. Finally, $\beta$ represents the regular home advantage while $\gamma$ indicates the additional home advantage of playing on an artificial pitch. Similarly, for team j playing at home against against team i it holds:

$$
y_{j i t}=\alpha_{j t}-\alpha_{i t}+\beta+d_{j} \gamma+\varepsilon_{j i t}
$$

where $d_{j}$ is an indicator for team $j$ playing on artificial grass. Since I ignore matches of teams who both have an artificial pitch, either $d_{i}$ is equal to zero or $d_{j}$ is equal to zero or they are both equal to zero.

To identify $\beta$ and $\gamma$, I make a couple of assumptions. First, I assume that the strength 
of a team is constant within a season. Of course, in reality the strength of a team may vary because of injuries or suspensions of key players. As long as the variation of strength within a season does not vary with the nature of the pitch there is no bias. I also assume that $\beta$ is the same for all teams. Finally, I assume that $\gamma$ is the same for all visiting teams who themselves play on natural grass and face a disadvantage when playing on an artificial pitch. Teams who play on an artificial pitch are assumed not to face a disadvantage when playing an natural grass.

By taking the average of equations (1) and (2), the strengths of both teams cancel out and for every pair of $i$ and $j$ in season $t$ it holds:

$$
S_{i j t}=\beta+d_{i j} \gamma+\epsilon_{i j t}
$$

where $S_{i j t}=\left(y_{i j t}+y_{j i t}\right) / 2, d_{i j}=\left(d_{j}+d_{i}\right) / 2, \epsilon_{i j t}=\left(\varepsilon_{i j t}+\varepsilon_{j i t}\right) / 2$. So for every pair of matches the sum of the match outcomes can be used as a dependent variable such that the constant in the equation is equal to the regular home advantage and the parameter $\gamma$ represents the additional home advantage of having an artificial pitch when playing against a team who has a natural grass home-ground. As shown by Clarke and Norman (1995) the point estimates of $\beta$ and $\gamma$ in equation (3) are identical to the calculations presented in Table 1.

Figure 2: Distribution goal differences (left) and point differences (right) by pairs of matches
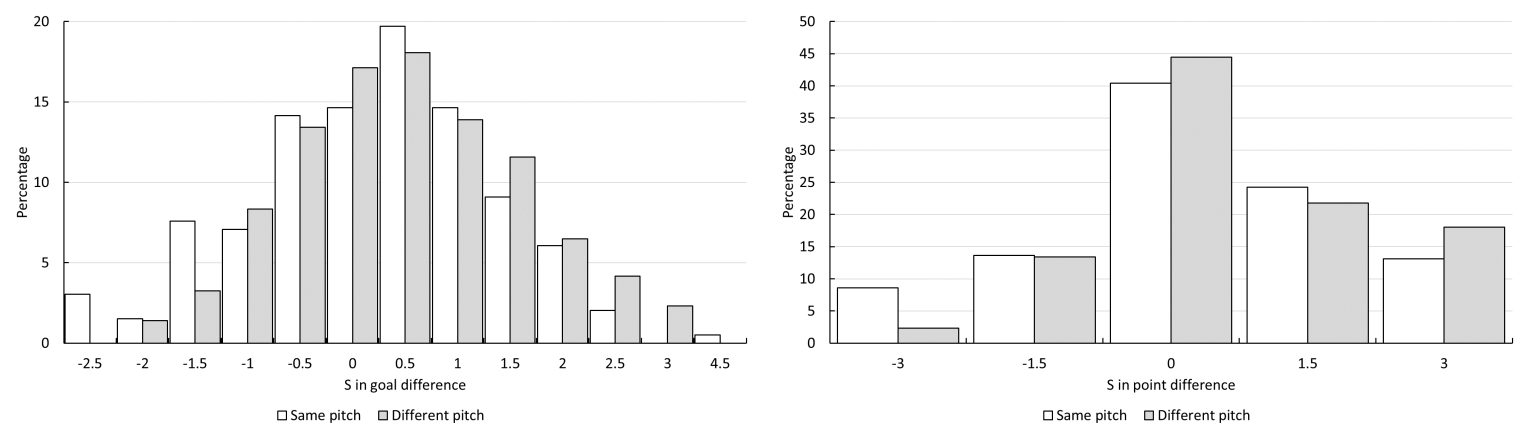

Note: Distribution of dependent variable S; see equation (3)

Because the dependent variable is specified for pairs of matches, the number of observations reduces from 828 to 414 . Figure 2 presents the distribution of the dependent variable where there is obviously more variation when the dependent variable is measured in goal difference. From both graphs, it is clear that the distribution of the dependent variable is shifted to the right when matches are played on different pitches as compared 
to when they are both played on natural grass.

Table 2: Parameter estimates baseline model

\begin{tabular}{llllll}
\hline & HA & \multicolumn{3}{c}{ AAP } & Obs. \\
\hline a. Goals per match & & & & & \\
\hline 1. Three seasons & 0.21 & $(0.08)^{* * *}$ & 0.53 & $(0.22)^{* *}$ & 414 \\
2. Season 2014-15 & 0.17 & $(0.14)$ & 0.43 & $(0.38)$ & 138 \\
3. Season 2015-16 & 0.26 & $(0.14)^{*}$ & 0.43 & $(0.39)$ & 138 \\
4. Season 2016-17 & 0.21 & $(0.14)$ & 0.73 & $(0.39)$ & 138 \\
5. Top 3 - 3 seasons & 0.26 & $(0.13)^{* *}$ & 0.69 & $(0.39)^{*}$ & 144 \\
6. Others - 3 seasons & 0.18 & $(0.11)$ & 0.52 & $(0.28)^{*}$ & 270 \\
7. Three seasons - all & 0.21 & $(0.08)^{* * *}$ & 0.53 & $(0.21)^{* *}$ & 459 \\
\hline b. Points per match & & & & & \\
\hline 1. Three seasons & 0.30 & $(0.12)^{* *}$ & 0.60 & $(0.31)^{*}$ & 414 \\
2. Season 2014-15 & 0.25 & $(0.19)$ & 0.52 & $(0.55)$ & 138 \\
3. Season 2015-16 & 0.36 & $(0.20)^{*}$ & 0.52 & $(0.55)$ & 138 \\
4. Season 2016-17 & 0.27 & $(0.20)$ & 0.75 & $(0.55)$ & 138 \\
5. Top 3 - 3 seasons & 0.25 & $(0.15)^{*}$ & 0.56 & $(0.47)$ & 144 \\
6. Others - 3 seasons & 0.33 & $(0.18)^{*}$ & 0.57 & $(0.42)$ & 270 \\
7. Three seasons - all & 0.28 & $(0.10)^{* * *}$ & 0.63 & $(0.29)^{* *}$ & 459 \\
\hline
\end{tabular}

Note: $\mathrm{HA}=$ regular home advantage; AAP $=$ extra advantage artificial pitch;

Obs. = Observations; in parentheses robust standard errors.

Table 2 shows the parameter estimates of equation (3). Panels a1 and b1 are identical to the calculations in Table 1 showing that the regular home advantage in goals is equal to 0.21 and in points equal to 0.30 , both significantly different from zero. The additional home advantage related to an artificial pitch is equal to 0.53 goals, significant at a $5 \%$-level and 0.60 points significant at a $10 \%$-level.

Panels a2 to a4 and b2 to b4 show parameter estimates separately by season. Although only a few parameter estimates are significantly different from zero, the point estimates are very similar to the main estimate. Apparently, there is a lot of random variation determining the outcome of matches and, therefore, it is difficult to get precise estimates of home advantage and additional home advantage of an artificial pitch. Panels a5, a6, b5 and b6 show parameter estimates for matches of the top-3 teams (Feyenoord, Ajax and PSV) and matches by the other teams. As indicated in Figure 1, by removing the top-3 teams from the analysis, there is an even distribution in terms of goals scored and goals conceded of teams with an artificial pitch and natural grass. Most of the estimated parameters are significantly different from zero with a magnitude that is similar to the baseline estimates. Panels a7 and b7 show the parameter estimates if I also include matches between teams who both have an artificial pitch. The results are basically the same as the baseline estimates of panel a1 and b1. 
To explore a possible reason for the presence of the home advantage, I introduce the average stadium attendance in the estimates, as an additive effect and as an interaction with the extra home advantage of an artificial pitch. The parameter estimates are presented in Table 3.

Table 3: Parameter estimates with stadium attendance

\begin{tabular}{lcccccccc}
\hline \multicolumn{3}{c}{ HA } & AAP & \multicolumn{3}{c}{ SA } & \multicolumn{3}{c}{ AAP*SA } \\
\hline a. Goals per match & & & & & & \\
\hline 1. & 0.18 & $(0.09)^{* *}$ & 0.68 & $(0.24)^{* * *}$ & 0.11 & $(0.07)$ & & \\
2. & 0.19 & $(0.09)^{* *}$ & 0.69 & $(0.24)^{* * *}$ & 0.12 & $(0.07)$ & 0.23 & $(0.29)$ \\
\hline b. Points per match & & & & & & \\
\hline 1. & 0.30 & $(0.13)^{* *}$ & 0.60 & $(0.35)^{*}$ & -0.00 & $(0.01)$ & & \\
2. & 0.30 & $(0.13)^{* *}$ & 0.60 & $(0.34)^{*}$ & -0.00 & $(0.10)$ & 0.03 & $(0.40)$ \\
\hline
\end{tabular}

Note: Based on 414 observations; $\mathrm{HA}=$ regular home advantage; AAP $=$ extra advantage artificial pitch; SA = demeaned average seasonal stadium attendance $(/ 10,000)$;

in parentheses robust standard errors.

Panel a of Table 3 presents the results for the goals per match and although the parameter estimate of stadium attendance is positive, suggesting that the home advantage is correlated with stadium attendance, the parameter estimate is not significantly different from zero. The same holds for the interaction between an artificial pitch and stadium attendance. Panel b shows that the results for points per match are similar. Clearly, the size of the home crowd has no effect on the magnitude of the home advantage nor on the additional home advantage of having an artificial pitch.

\section{Simulations}

The additional advantage of playing on an artificial pitch against a team that plays on natural grass is estimated to be equal to 0.6 points. Assuming that both teams are affected equally, but with an opposite sign, the team with an artificial pitch obtains 0.3 more points while the visiting team with a natural grass home pitch obtains 0.3 less points. As in every season each team with an artificial pitch played 12 of these matches they gained $12 * 0.3=3.6$ points while the teams with natural grass played 6 of these matches they lost $6^{*} 0.3=1.8$ points. At the top of the league all teams play on natural grass so each of them is affected similarly. At the bottom of the league this is different. Table 4 shows the results of simulations of the end-of-season number of points where the teams who play on a natural pitch are given 1.8 points extra while for the teams who play on an artificial pitch 3.6 points are subtracted. For each season, teams 
Table 4: Simulating the overall effects of playing on artificial pitches

\begin{tabular}{lccc|lccc|lccc}
\hline $2014-15$ & $\mathrm{P}$ & $\mathrm{PR}$ & $\mathrm{R}$ & 2015-16 & $\mathrm{P}$ & $\mathrm{PR}$ & $\mathrm{R}$ & $2016-17$ & $\mathrm{P}$ & $\mathrm{PR}$ & $\mathrm{R}$ \\
\hline PSV & 88 & 90 & 1 & PSV & 84 & 86 & 1 & Feyenoord & 82 & 84 & 1 \\
Ajax & 71 & 73 & 2 & Ajax & 82 & 84 & 2 & Ajax & 81 & 83 & 2 \\
AZ & 62 & 64 & 3 & Feyenoord & 63 & 65 & 3 & PSV & 76 & 78 & 3 \\
Feyenoord & 59 & 61 & 4 & AZ & 59 & 61 & 4 & Utrecht & 62 & 64 & 4 \\
Vitesse & 58 & 60 & 5 & FC Utrecht & 53 & 55 & 5 & Vitesse & 51 & 53 & 5 \\
PEC Zwolle & 53 & 49 & 7 & Heracles Almelo & 51 & 47 & 9 & AZ & 49 & 51 & 6 \\
sc Heerenveen & 50 & 52 & 6 & FC Groningen & 50 & 52 & 6 & FC Twente & 45 & 47 & 7 \\
FC Groningen & 46 & 48 & 8 & PEC Zwolle & 48 & 44 & 10 & FC Groningen & 43 & 45 & 8 \\
Willem II & 46 & 48 & 9 & Vitesse & 46 & 48 & 7 & sc Heerenveen & 43 & 45 & 9 \\
FC Twente & 43 & 45 & 10 & NEC & 46 & 48 & 8 & $\underline{\text { Heracles Almelo }}$ & 43 & 39 & 10 \\
FC Utrecht & 41 & 43 & 11 & ADO Den Haag & 43 & 39 & 13 & ADO Den Haag & 38 & 34 & 13 \\
SC Cambuur & 41 & 37 & 12 & sc Heerenveen & 42 & 44 & 11 & $\underline{\text { Excelsior }}$ & 37 & 33 & 14 \\
ADO Den Haag & 37 & 33 & 13 & FC Twente & 40 & 42 & 12 & Willem II & 36 & 38 & 11 \\
Heracles Almelo & 37 & 33 & 14 & Roda JC & 34 & 30 & 15 & $\underline{\text { PEC Zwolle }}$ & 35 & 31 & 15 \\
Excelsior & 32 & 28 & 17 & Excelsior & 30 & 26 & 16 & $\underline{\text { Sparta Rotterdam }}$ & 34 & 30 & 16 \\
NAC Breda & 28 & 30 & 15 & Willem II & 29 & 31 & 14 & N.E.C & 34 & 36 & 12 \\
Go Ahead Eagles & 27 & 29 & 16 & De Graafschap & 23 & 25 & 17 & $\underline{\text { Roda JC }}$ & 33 & 29 & 17 \\
FC Dordrecht & 20 & 16 & 18 & $\underline{\text { SC Cambuur }}$ & 18 & 14 & 18 & Go Ahead Eagles & 23 & 25 & 18 \\
\hline
\end{tabular}

Note: Simulations based on Table 2 panel b1; $\mathrm{P}=$ actual number of points end-of-season; PR =

(rounded) number of points removing the effects of artificial pitches; $\mathrm{R}=$ the ranking of the teams according to PR; the underlined teams have an artificial pitch; the teams in bold were relegated at the end of the season

are ordered according to the final ranking of the season. So, PSV won the championship in 2014/15 and 2015/16 and Feyenoord won the league in 2016/17. The bottom teams in each season were automatically relegated while some of the teams ending at places 16 and 17 were also relegated. The column labeled $\mathrm{P}$ shows the actual number of points, PR is the (rounded) number of points after the effects of playing on an artificial pitch are accounted for and $\mathrm{R}$ is the ranking of the teams according to PR.

Clearly, the bottom teams remain the bottom teams but some of the teams that relegated would not have been relegated if it had not been for artificial pitches. NAC Breda would have ended as number 15 in 2014-15, while NEC would have ended as number 12 in 2016-17. Both teams would have not been relegated in these seasons. Excelsior (in 2014-15 and 2015-16) and Sparta Rotterdam (in 2016-17) would have been exposed to promotion-relegation play-off matches against teams from the second level. So, rather than staying in the top tier for sure, they could have been relegated.

\section{Conclusion}

Home advantage is a phenomenon that is undisputed in professional football and not much of an issue as in all competitions teams play an equal number of home and away matches. However, from an analysis of matches in three seasons of professional football in 
the Netherlands, it appears that teams who play on an artificial pitch have an additional home advantage compared to teams who play on natural grass. For the top teams, this does not matter since they all have a natural grass home pitch. Therefore, they face the same disadvantage and the ranking at the end of the season will not change because they have to play some away matches on an artificial pitch. At the bottom of the league this is different. Teams playing near the bottom on natural grass have been regulated who would have otherwise stayed in the top tier. Similarly, teams playing their home matches on an artificial pitch stayed up but could have otherwise been relegated. Clearly, artificial pitches are a reason for concern as they introduce an unfair home advantage for some teams in an otherwise highly competitive sport.

\section{References}

Andersson, H., Ekblom, B., and Krustrup, P. (2008). Elite football on artificial turf versus natural grass: Movement patterns, technical standards, and player impressions. Journal of Sports Sciences, 26(2):113-122.

Barnett, V. and Hilditch, S. (1993). The effect of an artificial pitch surface on home teamperformance in football (soccer). Journal of the Royal Statistical Society: Series A (Statistics in Society), 156(1):39-50.

Clarke, S. R. and Norman, J. M. (1995). Home ground advantage of individual clubs in English soccer. The Statistician, 44(4):509-521.

Ekstrand, J., Hägglund, M., and Fuller, C. W. (2011). Comparison of injuries sustained on artificial turf and grass by male and female elite football players. Scandinavian Journal of Medicine Es Science in Sports, 21:824-832.

Hvattum, L. M. (2015). Playing on artificial turf may be an advantage for Norwegian soccer teams. Journal of Quantitative Analysis in Sports, 11(3):183-192.

Lanzetti, R. M., Ciompi, A., Lupariello, D., Guzzini, M., De Carli, A., and Ferretti, A. (2017). Safety of thid-generation artificial turf in male elite professional soccer players in Italian major league. Scandinavian Journal of Medicine E Science in Sports, 27:435-439.

Pollard, R. (2006). Worldwide regional variations in home advantage in association football. Journal of Sports Sciences, 24(3):231-240.

Pollard, R. and Armatas, V. (2017). Factors affecting home advantage in football World Cup qualification. International Journal of Performance Analysis in Sport, 17(1-2):121-135.

Ponzo, M. and Scoppa, V. (2017). Does the home advantage depend on crowd support? Evidence from same-stadium derbies. Journal of Sports Economics, page forthcoming.

Trombley, M. J. (2016). Does artificial grass affect the competitive balance in major league soccer? Journal of Sports Analytics, 2:73-87. 